\title{
FEA Versus IGA in a Two-Node Beam Element Based on Unified and Integrated Method
}

\author{
Andi Makarim Katili and Irwan Katili* \\ Universitas Indonesia, Civil Engineering Department, Depok 16424, Indonesia
}

Received 2 December 2018; Accepted (in revised version) 14 July 2019

\begin{abstract}
This paper presents a new concept called Unified and Integrated Method for a shear deformable beam element. In this method, Timoshenko beam theory is unified and integrated in such a way that takes into account the effect of transverse shear and maintains the shear locking free condition at the same time to generate proper behavior in the analysis of thin to thick beams. The unified and integrated method is applied to finite element analysis (FEA) and isogeometric analysis (IGA) on two-node beam element. This method will be used to analyze uniformly loaded beams with various boundary conditions. A shear influence factor of $\phi$, which is a function of beam thickness ratio $(L / h)$, is expressed explicitly as control of the transverse shear strain effect. The analysis gives interesting results showing that applying the unified and integrated method in FEA and IGA will yield exact values of DOF's and displacement function even when using only a single element. Numerical examples demonstrate the validity and efficiency of the unified and integrated methods.
\end{abstract}

AMS subject classifications: 65L60, 74-10, 74K10, 74S05, 74S22

Key words: Finite element analysis, isogeometric analysis, unified and integrated method, timoshenko beam theory.

\section{Introduction}

Bernoulli-Euler beam theory, also known as classical beam theory, was introduced by Daniel Bernoulli and Leonhard Euler around 1750. It has been widely used to analyze the behavior of the bending element because of its simplicity. The theory assumes that after deformation cross-sections remain plane and orthogonal to the beam axis and that deformation slopes are small. It suggests that shear deformation $\gamma$ is neglected and rotation $\theta$ is equal to the derivative of deflection. Hence, it is more suitable for a slender beam. Vertical deflection $v$ is the only unknown variable is this theory. The curvature in

*Corresponding author.

Email: irwan.katili@eng.ui.ac.id (I. Katili) 
the Principal of Virtual Work (PVW) is expressed by the second derivatives of $v(x)$ as the only deformation. This theory requires $C^{1}$ continuity to ensure a smooth deflection field.

Timoshenko beam theory $[1,2]$ developed later in the early 20th century offers an improvement. Unlike the classical theory, it takes into account of shear deformation and rotational bending effects so that the previously perpendicular plane sections will not necessarily remain perpendicular to the beam axis after deformation. In this theory, deflection $(v)$ and rotation $(\theta)$ are independent of each other. The development of Timoshenko beam element is simpler than of Bernoulli-Euler beam element as it requires $C^{0}$ continuity for the deflection and rotation fields. However, the 2-noded Timoshenko beam element suffers from a phenomenon called shear locking when analyzing thin beams. They only provide reasonable solutions in the cases of a thick beam, but give unrealistically stiffer results for thin beams $(L / h>20)$. This phenomenon disqualifies Timoshenko beam elements for the analysis of slender beams.

A popular method to alleviate shear locking in Timoshenko beam elements is by under-integrating the terms in shear stiffness using a quadrature of one order less than needed for exact integration. This method reduces the effect of the transverse shear stiffness and yields constant transverse shear strains along the beam. The terms in bending stiffness are still integrated exactly. This method is known as Selective Reduced Integration (SRI).

There are many methods to eliminate shear locking, one of which is the Assumed Natural Strain (ANS). A number of Timoshenko beam elements have adopted the ANS to deal with the shear locking problem. By applying the ANS, the transverse shear strain in a beam element with two nodes and two degrees of freedom per node becomes constant along with the element [3]. The two-node element with linear interpolation demonstrates satisfactory outcomes over a wide variety of length to thickness ratio. Yet, in the matter of convergence speed, it cannot be compared with the Bernoulli-Euler element, which neglects the shear deformation.

While developing Discrete Shear Gap (DSG) method to overcome shear locking, Bletzinger et al. [4] also applied the ANS concept. DSG beam element satisfies the kinematic equation for the shear strains at discrete nodes and significantly reduces the shear strains. The key of the DSG method is calculating the discrete shear gap at nodes and interpolating them across the element domain. Just like reduced integration, the application of the DSG concept in the beam element with 2 nodes gives a constant shear along the beam.

It is well known that it is possible to derive a 2 nodes beam element that gives exact results (at least at nodes) based on mixed formulation [5]. Exact here means that the results are valid for thick to thin beams, without the occurrence of shear locking.

Another beam element that adopts the ANS concept is Discrete Shear Beam (DSB). DSB element [6] uses cubic interpolations to calculate total vertical displacement $(v)$ and quadratic interpolations to calculate rotation $(\theta)$. In this element, the transverse shear strain is defined as constant along the beam by using the discrete shear method. DSB element has been the basis of the development of triangular DKMT and quadrilateral DKMQ plate and shell elements [7-17]. However, besides the good performance over 
thin to thick beam problems, it only provides exact solutions at nodes.

Recently, a new approach to deal with the shear locking phenomenon in beam and bending plate problems has been proposed $[6,18-24,26]$. By modifying the Timoshenko beam and the Reissner-Mindlin plate theory, this approach has created a strong interdependence between the bending displacement and rotation which prevents the shear locking from occurring to occur in beam and plate problems, respectively.

Kiendl et al. [21] developed a unified approach in an isogeometric analysis for shear deformable beams. In this approach, the vertical displacement $(v)$ is divided into two parts, i.e., the bending part $\left(v_{b}\right)$ and shear part $\left(v_{s}\right)$. The approach shows a strong correlation between the two parts, allowing that all derived variables to be expressed in terms of the bending displacement $\left(v_{b}\right)$ as the only variable. Actually, the same idea of dividing the vertical displacement equation was suggested quite a long time ago in the early days of finite beam elements by Kapur [22], $\mathrm{Li}$ [23] and Falsone et al. [24] with a similar approach.

Recently Katili et al. [26] published the application of a 2-node beam element using a unified and integrated approach in functionally graded materials and considering a coupling axial-bending effect due to unsymmetrical material layer.

This article compares the results of applying the unified and integrated method into isogeometric and finite element analysis on two-node beam element problems. The organization of the article is as follows. First, it presents the limitation of the classical and Timoshenko beam theory. It is followed by the explanation of several elements that used ANS to cope with the shear locking phenomenon and a brief introduction of the unified approach that can eliminate the shear locking. In Section 2, the development of a unified and integrated method is described. It shows how to integrate and unify all equations in Timoshenko beam theory, resulting in similar equations with Bernoulli theory. Section 3 will present the formulation of the Unified and Integrated (UI) beam element. The formulation of IGA Galerkin for Timoshenko beam will be presented in Section 4. The performance of the UI beam and IGA Galerkin will be compared in Section 5. Conclusions, acknowledgements, and references are given in Section 6.

\section{Unified and integrated method}

To establish notation and convention, the equations for Timoshenko beams are first summarized below. Consider a Timoshenko beam $[1,2]$ with the beam axis $x$ of the local Cartesian coordinate orientated in the axial direction coinciding with the neutral axis. The positive beam axis $y$ orientated up orthogonal to the $x$-axis.

Assuming that displacement is small, and shear deformation $\gamma$ is uniform at any cross-section and only dependent on $x$, the curvature and shear deformation at any point $x$ along the beam is given by:

$$
\chi=-\frac{d \theta_{b}}{d x}, \quad \gamma=\frac{d v}{d x}-\theta_{b}
$$


Where $v(x)=$ vertical displacement in $y$ direction, $\theta_{b}(x)=$ bending rotation, $\chi(x)=$ curvature, and $\gamma(x)=$ transverse shear deformation.

The constitutive laws for axial stresses is:

$$
\sigma=y E \chi
$$

The relation between shear stress and shear strain is:

$$
\tau=G \gamma,
$$

where $E$ is the Young modulus of elasticity, $G$ is the shear modulus where $G=E / 2(1+v)$, $v$ is the Poisson's ratio.

The stress resultants $M$ and $T$ are integral of the stress component $\sigma$ and $\tau$, respectively, as follows:

$$
M=\iint_{A} y \sigma d A, \quad T=\iint_{A} \tau d A .
$$

The constitutive equations for the bending moments and shear forces are obtained from (2.1)-(2.4) and given as follows:

$$
M=E I \chi, \quad T=\kappa G A \gamma,
$$

where $E I$ is the bending rigidity, $\kappa G A$ is the shear rigidity, $\kappa$ is the shear correction factor.

The equilibrium equations for Timoshenko beam:

$$
\begin{aligned}
& \frac{d M}{d x}=T \\
& \frac{d T}{d x}=f .
\end{aligned}
$$

Where $f$ is the uniform load per unit length.

By substituting Eq. (2.1) and (2.5) into Eq. (2.6a) and (2.6b), we get the differential equations for the exact solution of Timoshenko beam in term of $v$ and $\theta_{b}$ :

$$
\begin{aligned}
& E I \frac{d^{2} \theta_{b}}{d x^{2}}+\kappa G A\left(\frac{d v}{d x}-\theta_{b}\right)=0, \\
& \kappa G A\left(\frac{d^{2} v}{d x^{2}}-\frac{d \theta_{b}}{d x}\right)=f .
\end{aligned}
$$

As can be seen in $[21,22]$, these last two differential equations can be combined into a single equation with $\theta_{b}$ as the only unknown variable

$$
-E I \frac{d^{3} \theta_{b}}{d x^{3}}=f .
$$


By integrating Eq. (2.7a), we find the vertical displacement $v$ as follows:

$$
v=\int_{0}^{x} \theta_{b} d x-\frac{E I}{\kappa G A} \frac{d \theta_{b}}{d x}+c .
$$

Where $c$ is an integration constant.

In Eq. (2.9) the total vertical displacement is divided into two parts: first $v_{b}$ as a bending displacement and second $v_{s}$ as a shear displacement as follows:

$$
\begin{aligned}
& v=v_{b}+v_{s}, \\
& v_{b}=\int_{0}^{x} \theta_{b} d x+c, \quad v_{s}=-\frac{E I}{\kappa G A} \frac{d \theta_{b}}{d x} .
\end{aligned}
$$

Differentiating Eq. (2.10a) yields:

$$
\begin{aligned}
& \frac{d v}{d x}=\frac{d v_{b}}{d x}+\frac{d v_{s}}{d x}, \\
& \frac{d v_{b}}{d x}=\theta_{b}, \\
& \frac{d v_{s}}{d x}=\theta_{s}=-\frac{E I}{\kappa G A} \frac{d^{2} \theta_{b}}{d x^{2}}=-\frac{E I}{\kappa G A} \frac{d^{3} v_{b}}{d x^{3}} .
\end{aligned}
$$

Substituting (2.11b) into curvature in Eq. (2.1) yields:

$$
\chi=-\frac{d^{2} v_{b}}{d x^{2}} .
$$

Substituting (2.10b)-(2.11b) into shear deformation in Eq. (2.1) and from (2.11c), we obtain:

$$
\gamma=\theta_{s}=\frac{d v_{s}}{d x}=-\frac{E I}{\kappa G A} \frac{d^{2} \theta_{b}}{d x^{2}}=-\frac{E I}{\kappa G A} \frac{d^{3} v_{b}}{d x^{3}} .
$$

By integrating (2.13) we obtain:

$$
v_{s}=-\frac{E I}{\kappa G A} \frac{d^{2} v_{b}}{d x^{2}}=\frac{E I}{\kappa G A} \chi .
$$

Finally, Eq. (2.10a) can be expressed in terms of $v_{b}$ only:

$$
v=v_{b}-\frac{E I}{\kappa G A} \frac{d^{2} v_{b}}{d x^{2}} .
$$

Substituting Eq. (2.11b) into (2.8) we obtain:

$$
-E I \frac{d^{4} v_{b}}{d x^{4}}=f .
$$


These differential equations (2.12)-(2.16) are similar to the ones for a Bernoulli-Euler beam theory with $v$ replacing $v_{b}$, but have accounted shear deformation. In fact, for very slender beams, where $\frac{E I}{\kappa G A} \rightarrow 0$, the equations will show that $v_{s} \rightarrow 0$ and $v_{b} \rightarrow v$, confirming that Eqs. (2.12)-(2.16) are identical to the Bernoulli-Euler equations. Four boundary conditions are needed to complete the fourth order differential equation (2.16).

The beam boundaries are denoted by $\Gamma=\{0\} U\{L\}$, with $L$ is the length of the beam. Furthermore, $\Gamma_{v}, \Gamma_{\theta}, \Gamma_{M}, \Gamma_{T}$ indicate the boundaries with prescribed $v, \theta, M$ and $T$, respectively. The boundary condition are formulated as follows,

$$
\begin{aligned}
& \bar{v}=v_{b}-\frac{E I}{\kappa G A} \frac{d^{2} v_{b}}{d x^{2}} \rightarrow \Gamma_{v}, \\
& \bar{\theta}_{b}=\frac{d v_{b}}{d x} \rightarrow \Gamma_{\theta_{b}}, \\
& \bar{M}= \pm-E I \frac{d^{2} v_{b}}{d x^{2}} \rightarrow \Gamma_{M}, \\
& \bar{T}=-E I \frac{d^{3} v_{b}}{d x^{3}} \rightarrow \Gamma_{T} .
\end{aligned}
$$

The barred symbols designate the imposed boundary values.

Take notice that a zero-vertical displacement at boundary condition means that the sum of $v_{b}$ and $v_{s}$ is zero, i.e., $v_{b}+v_{s}=0 \rightarrow v_{b} \neq 0, v_{s} \neq 0$, not that both $v_{b}$ and $v_{s}$ are zero at the boundary.

The classical of the principle of virtual work (PVW) takes the form:

$$
\int_{0}^{L} \chi^{*} E I \chi d x+\int_{0}^{L} \gamma^{*} \kappa G A \gamma d x=f \int_{0}^{L} v^{*} d x+\left.\left(\theta_{b}^{*}\right)\right|_{\Gamma_{M}} \bar{M}+\left.\left(v^{*}\right)\right|_{\Gamma_{T}} \bar{T} .
$$

The internal virtual work, consisting of bending and shear parts is on the left side of the equation. The external virtual work is on the right side. As a convention, concentrated point load $\bar{T}$ and distributed loads $f$, acting in the direction of the global $y$-axis are taken as positive. Consistent with the definition of the rotation, the concentrated moments acting at beam points are taken as positive if they act anticlockwise.

\section{UI element based on unified and integrated method}

The 2-node UI element is formulated based on an unified and integrated method (2.12)(2.18). The element has six degrees of freedom (DOFs), e.g., $v_{b_{i}}, v_{s_{i}}, \theta_{i}$ at each node $i$ as shown in Fig. 1. The only unknown variable in the UI element equation is bending displacement $v_{b}$, which is approximated by using a 5 th-degree polynomial expansion. To ensure a smooth bending displacement, slopes and curvatures have to be continuous across adjacent elements. Therefore, $C^{2}$ Hermite shape functions are required. The PVW in this element involves the second derivatives of $v_{b}(x)$. The 5 th-degree polynomial for 


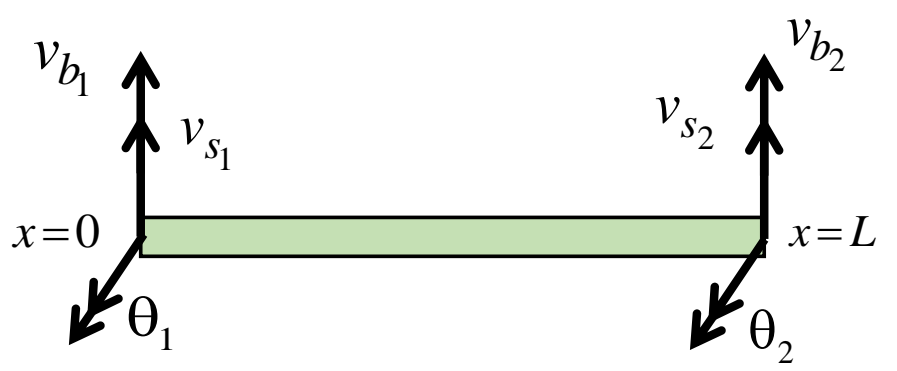

Figure 1: Degree of freedom of UI beam element.

bending displacement is:

$$
v_{b}=\langle P\rangle\left\{a_{n}\right\}=\left\langle 1 \quad x \quad x^{2} \quad x^{3} \quad x^{4} \quad x^{5}\right\rangle\left\{a_{n}\right\} .
$$

From (2.11b) the rotational function is:

$$
\theta_{b}=\frac{d v_{b}}{d x}=\langle P, x\rangle\left\{a_{n}\right\}=\left\langle\begin{array}{llllll}
0 & 1 & 2 x & 3 x^{2} & 4 x^{3} & 5 x^{4}
\end{array}\right\rangle\left\{a_{n}\right\} .
$$

From (2.14) the shear displacement function is:

$$
v_{s}=-\frac{E I}{\kappa G A}\left\langle P_{, x x}\right\rangle\left\{a_{n}\right\}=-\frac{E I}{\kappa G A}\left\langle\begin{array}{llllll}
0 & 0 & 2 & 6 x & 12 x^{2} & \left.20 x^{3}\right\rangle\left\{a_{n}\right.
\end{array} .\right.
$$

We introduce shear influence factor:

$$
\phi=\frac{E I}{\kappa G A} \frac{12}{L^{2}} .
$$

And (3.3) become:

$$
v_{s}=-\frac{\phi L^{2}}{12}\left\langle P_{, x x}\right\rangle\left\{a_{n}\right\}=-\frac{\phi L^{2}}{12}\left\langle\begin{array}{llllll}
0 & 0 & 2 & 6 x & 12 x^{2} & \left.20 x^{3}\right\rangle\left\{a_{n}\right.
\end{array} .\right.
$$

Where

$$
\left\{a_{n}\right\}=\left\langle a_{n}\right\rangle^{T}=\left\langle\begin{array}{llllll}
a_{1} & a_{2} & a_{3} & a_{4} & a_{5} & a_{6}
\end{array}\right\rangle^{T} .
$$

By substituting the conditions of each node:

$$
\left\{\begin{array}{c}
v_{b_{1}} \\
v_{S_{1}} \\
\theta_{1} \\
v_{b_{2}} \\
v_{s_{2}} \\
\theta_{2}
\end{array}\right\}=\left[\begin{array}{cccccc}
1 & 0 & 0 & 0 & 0 & 0 \\
0 & 0 & -2 \frac{\phi L^{2}}{12} & 0 & 0 & 0 \\
0 & 1 & 0 & 0 & 0 & 0 \\
1 & L & L^{2} & L^{3} & L^{4} & L^{5} \\
0 & 0 & -2 \frac{\phi L^{2}}{12} & -6 \frac{\phi L^{2}}{12} L & -12 \frac{\phi L^{2}}{12} L^{2} & -20 \frac{\phi L^{2}}{12} L^{3} \\
0 & 1 & 2 L & 3 L^{2} & 4 L^{3} & 5 L^{4}
\end{array}\right]\left\{\begin{array}{c}
a_{1} \\
a_{2} \\
a_{3} \\
a_{4} \\
a_{5} \\
a_{6}
\end{array}\right\}
$$






Figure 2: Shape functions of UI beam element.

or

$$
\left\{u_{n}\right\}=\left[P_{n}\right]\left\{a_{n}\right\} \rightarrow\left\{a_{n}\right\}=\left[P_{n}\right]^{-1}\left\{u_{n}\right\} .
$$

Then, by substituting (3.7)-(3.8) into (3.1), we get

$$
\begin{aligned}
& v_{b}=\langle N\rangle\left\{u_{n}\right\}, \\
& \langle N\rangle=\left\langle\begin{array}{lllllll}
N_{v_{b_{1}}} & N_{v_{s_{1}}} & N_{\theta_{1}} & N_{v_{b_{2}}} & N_{v_{s_{1}}} & N_{\theta_{2}}
\end{array}\right\rangle, \\
& \left\{u_{n}\right\}=\left\langle u_{n}\right\rangle^{T}=\left\langle\begin{array}{lllllll}
v_{b_{1}} & v_{s_{1}} & \theta_{1} & v_{b_{2}} & v_{s_{2}} & \theta_{2}
\end{array}\right\rangle .
\end{aligned}
$$

Where $\left\{u_{n}\right\}$ is the nodal displacement $v_{b_{1}}, v_{s_{1}}, \theta_{1}$ and $v_{b_{2}}, v_{s_{2}}, \theta_{2}$ are the bending displacement, shear displacement and the rotation of nodes 1 and 2 , respectively. $\langle N\rangle$ are the $C^{2}$ Hermite shape functions

$$
\begin{cases}\text { Node } 1 & \text { Node 2 } \\ N_{v_{b_{1}}}=\frac{1}{L^{5}}(L-x)^{3}\left(L^{2}+3 L x+6 x^{2}\right) & N_{v_{b_{2}}}=\frac{x^{3}}{L^{5}}\left(10 L^{2}-15 L x+6 x^{2}\right) \\ N_{v_{s_{1}}}=-\frac{1}{\phi L^{5}} 6 x^{2}(L-x)^{3} & N_{v_{s_{2}}}=-\frac{6 x^{3}}{\phi L^{5}}(L-x)^{2} \\ N_{\theta_{1}}=\frac{1}{L^{4}} x(L+3 x)(L-x)^{3} & N_{\theta_{2}}=\frac{x^{3}}{L^{4}}\left(-4 L^{2}+7 L x-3 x^{2}\right)\end{cases}
$$

The shape functions (3.10) shows that $N_{v_{b 1}}$ and $N v_{b 2}$ take a unit value at a node and zero at the other node (see Fig. 2), and their first derivatives are zero at both nodes, while the opposite occurs with $N_{\theta_{1}}$ and $N_{\theta_{2}}$. 
Using Eq. (2.12), the curvature at a point within the element is obtained in terms of the nodal DOFs by

$$
\chi=-\frac{d^{2} v_{b}}{d x^{2}}=\left\langle B_{b}\right\rangle\left\{u_{n}\right\}
$$

where

$$
\left\langle B_{b}\right\rangle=-\left\langle\begin{array}{llllll}
N_{v_{b_{1}}}, x x & N_{v_{s_{1}}, x x} & N_{\theta_{1}, x x} & N_{v_{b_{2}}, x x} & N_{v_{s_{2}}, x x} & N_{\theta_{2}, x x}
\end{array} .\right.
$$

Using Eq. (2.13), the shear deformation at a point within the element is obtained in terms of the nodal DOFs by

$$
\gamma=-\frac{E I}{\kappa G A} \frac{d^{3} v_{b}}{d x^{3}}=\left\langle B_{s}\right\rangle\left\{u_{n}\right\}
$$

where

$$
\left\langle B_{s}\right\rangle=-\frac{\phi L^{2}}{12}\left\langle\begin{array}{llllll}
N_{v_{b_{1}}}, x x x & N_{v_{s_{1}}}, x x x & N_{\theta_{1}}, x x x & N_{v_{b_{2}}}, x x x & N_{v_{s_{2}}}, x x x & N_{\theta_{2}, x x x}
\end{array}\right\rangle .
$$

By substituting (3.11)-(3.14) into (2.18), the PVW for an individual element can be written as

$$
W^{e}=\left\langle u_{n}^{*}\right\rangle\left([k]\left\{u_{n}\right\}-\left\{f_{n}\right\}\right)
$$

After simplifying the virtual displacements, the total element stiffness matrix:

$$
[k]=\left[k_{b}\right]+\left[k_{s}\right], \quad\left[k_{b}\right]=E I \int_{0}^{L}\left\{B_{b}\right\}\left\langle B_{b}\right\rangle d x, \quad\left[k_{s}\right]=\kappa G A \int_{0}^{L}\left\{B_{s}\right\}\left\langle B_{s}\right\rangle d x .
$$

Where $\left[k_{b}\right],\left[k_{s}\right]$ and $\left\{f_{n}\right\}$ are the bending stiffness matrix, the shear stiffness matrix and the equivalent nodal force vector for the element, respectively. Splitting the element stiffness matrix as in (3.16) is more convenient as it allows us to identify the bending and shear contributions.

The bending stiffness for UI element is expressed by:

$$
\left[k_{b}\right]=\left[\begin{array}{cccccc}
\frac{120 E I}{7 L^{3}} & -\frac{3 \kappa G A}{7 L} & \frac{60 E I}{7 L^{2}} & -\frac{120 E I}{7 L^{3}} & \frac{3 \kappa G A}{7 L} & \frac{60 E I}{7 L^{2}} \\
-\frac{3 \kappa G A}{7 L} & \frac{3(\kappa G A)^{2} L}{35 I I} & -\frac{11 \kappa G A}{35} & \frac{3 \kappa G A}{7 L} & \frac{(\kappa G A)^{2} L}{70 E I} & -\frac{4 \kappa G A}{35} \\
\frac{60 E I}{7 L^{2}} & -\frac{11 \kappa G A}{35} & \frac{192 E I}{35 L} & -\frac{60 E I}{7 L^{2}} & \frac{4 \kappa G A}{35} & \frac{108 E I}{35 L} \\
-\frac{120 E I}{7 L^{3}} & \frac{3 \kappa G A}{7 L} & -\frac{60 E I}{7 L^{2}} & \frac{120 E^{2}}{7 L^{3}} & -\frac{3 \kappa G A}{7 L} & -\frac{60 E I}{7 L^{2}} \\
\frac{3 \kappa G A}{7 L} & \frac{(\kappa G A)^{2} L}{70 I} & \frac{4 \kappa G A}{35} & -\frac{3 \kappa G A}{7 L} & \frac{3(\kappa G A)^{2} L}{35} & \frac{11 \kappa G A}{35} \\
\frac{60 E I}{7 L^{2}} & -\frac{4 \kappa G A}{35} & \frac{108 E I}{35 L} & -\frac{60 E I}{7 L^{2}} & \frac{11 \kappa G A}{35} & \frac{192 E I}{35 L}
\end{array}\right] .
$$


The shear stiffness for UI element is expressed by:

$$
\left[k_{s}\right]=\phi\left[\begin{array}{rrrrrr}
\frac{60 E I}{L^{3}} & -\frac{5 \kappa G A}{L} & \frac{30 E I}{L^{2}} & -\frac{60 E I}{L^{3}} & \frac{5 \kappa G A}{L} & \frac{30 E I}{L^{2}} \\
-\frac{5 \kappa G A}{L} & \frac{3(\kappa G A)^{2} L}{4 E I} & -3 \kappa G A & \frac{5 \kappa G A}{L} & -\frac{(\kappa G A)^{2} L}{4 E I} & -2 \kappa G A \\
\frac{30 E I}{L^{2}} & -3 \kappa G A & \frac{16 E I}{L} & -\frac{30 E I}{L^{2}} & 2 \kappa G A & \frac{14 E I}{L} \\
-\frac{60 E I}{L^{3}} & \frac{5 \kappa G A}{L} & -\frac{30 E I}{L^{2}} & \frac{60 E I}{L^{3}} & -\frac{5 \kappa G A}{L} & -\frac{30 E I}{L^{2}} \\
\frac{5 \kappa G A}{L} & -\frac{(\kappa G A)^{2} L}{4 E I} & 2 \kappa G A & -\frac{5 \kappa G A}{L} & \frac{3(\kappa G A)^{2} L}{4 E I} & 3 \kappa G A \\
\frac{30 E I}{L^{2}} & -2 \kappa G A & \frac{14 E I}{L} & -\frac{30 E I}{L^{2}} & 3 \kappa G A & \frac{16 E I}{L}
\end{array}\right] .
$$

The UI element stiffness matrix is obtained from the sum of the bending and shear stiffness matrix:

$$
[k]=\left[k_{b}\right]+\left[k_{s}\right] .
$$

Shear influence factor $\phi$ depends on the geometry and the material properties of the transverse cross-section. A small value of $\phi$ indicates that shear strain effects can be neglected. Transverse shear effect is negligible for a slender beam. For slender beams $\phi \rightarrow 0$, $\gamma$ and shear stiffness $\left[k_{s}\right]$ in (3.18) should disappear, meaning that for slender beams, the 2-node UI element can deliver the same solution as given by the Euler-Bernoulli beam theory.

For a uniform load $f_{0}$, the external energy is given as follows:

$$
\begin{aligned}
\Pi_{e x t} & =\int_{0}^{L} f_{0} v(x) d x=f_{0} \int_{0}^{L}\left(v_{b}(x)+v_{s}(x)\right) d x \\
& =f_{0} \int_{0}^{L}\left(v_{b}-\frac{L^{2} \phi}{12} \frac{d^{2} v_{b}}{d x^{2}}\right) d x=\left\langle u_{n}\right\rangle\left\{f_{n}\right\} .
\end{aligned}
$$

Where equivalent nodal force vector can be written as:

$$
\begin{aligned}
& \left\{f_{n}\right\}=\left\langle f_{n}\right\rangle^{T}=\left\langle\begin{array}{llllll}
f_{v_{b 1}} & f_{v_{s 1}} & f_{\theta_{1}} & f_{v_{b 2}} & f_{v_{s 2}} & f_{\theta_{2}}
\end{array}\right\rangle^{T}, \\
& \left\langle f_{n}\right\rangle=f_{0} L\left\langle\frac{1}{2} \frac{L(6+5 \phi)}{60}-\frac{L^{2}}{120} \quad \frac{1}{2} \quad-\frac{L(6+5 \phi)}{60}-\frac{L^{2}}{120}\right\rangle .
\end{aligned}
$$

\section{IGA Galerkin based on unified and integrated beam method}

In isogeometric analysis, the B-spline functions are not only used to describe the geometric entities but also to approximate the solution in an isoparametric fashion. Control point variables function as degrees of freedom and the knot spans represent elements.

\subsection{B-Spline basis functions}

In one-dimensional case, B-Spline basis functions are determined using the Cox-de Boor [?] recursion formulas. The construction of the B-Spline basis functions are generated from 


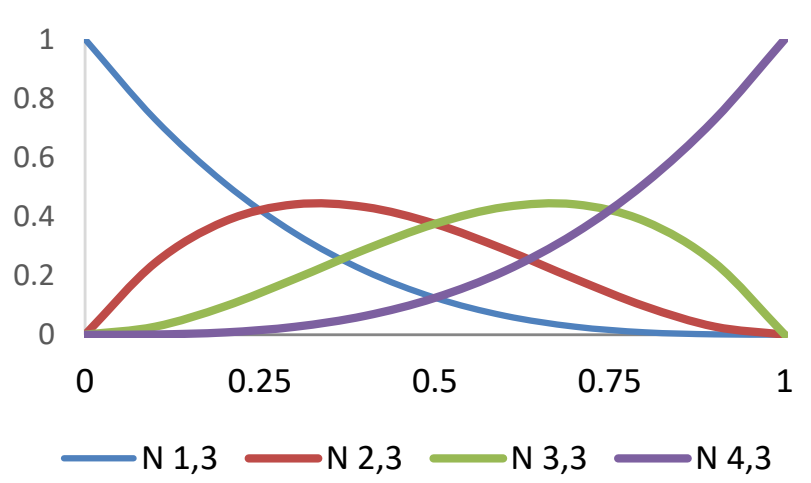

Figure 3: Cubic B-spline basis functions generated from the open knot vector $\Xi_{\xi}=[0,0,0,0,1,1,1,1]$.

the so-called knot vectors $\Xi_{\xi}$ and are defined in a parametric space $\xi \in[0,1]$ into a set of intervals as follows:

$$
\Xi_{\xi}=\left[\xi_{1}=0, \xi_{2}, \cdots, \xi_{n+p+1}=1\right] .
$$

Where $p$ represents the polynomial degree order, while $n$ represents the number of basis functions. The interval $\left[\xi_{i}, \xi_{i+1}\right]$ is called a knot span, and the interval $\left[\xi_{1}, \xi_{n+p+1}\right]$ is called a patch. The knot vector $\Xi_{\xi}$ is called open if the first and the last knots are repeated $(p+1)$ times. We start with piecewise constants

$$
N_{i, 0}(\xi)=\left\{\begin{array}{ll}
1, & \text { if } \xi_{i} \leq \xi<\xi_{i+1}, \\
0, & \text { otherwise, }
\end{array} \text { for } p=0\right.
$$

Linear, quadratic and higher order functions are defined by

$$
N_{i, p}(\xi)=\frac{\xi-\xi_{i}}{\xi_{i+p}-\xi_{i}} N_{i, p-1}(\xi)+\frac{\xi_{i+p+1}-\xi}{\xi_{i+p+1}-\xi_{i+1}} N_{i+1, p-1}(\xi) \text { for } p \geq 1,
$$

$\xi_{i}$ are coordinates of the knots in the parametric space, collected in a knot vector $\Xi_{\xi}$.

Basis functions for $p=3$ and $N E L T=1$, knot vectors $\Xi_{\xi}=[0,0,0,0,1,1,1,1]$,

$$
\begin{array}{ll}
N_{1}(\xi)=N_{1,3}(\xi)=(1-\xi)^{3}, & N_{2}(\xi)=N_{2,3}(\xi)=3 \xi(1-\xi)^{2}, \\
N_{3}(\xi)=N_{3,3}(\xi)=3 \xi^{2}(1-\xi), & N_{4}(\xi)=N_{4,3}(\xi)=\xi^{3} .
\end{array}
$$

An example of a set of cubic B-Spline functions generated from an open knot vector is illustrated in Fig. 3.

Basis functions for $p=5$ and $N E L T=1$, knot vectors $\Xi_{\xi}=[0,0,0,0,0,0,1,1,1,1,1,1]$

$$
\begin{array}{ll}
N_{1}(\xi)=N_{1,5}(\xi)=(1-\xi)^{5}, & N_{2}(\xi)=N_{2,5}(\xi)=5 \xi(1-\xi)^{4}, \\
N_{3}(\xi)=N_{3,5}(\xi)=10 \xi^{2}(1-\xi)^{3}, & N_{4}(\xi)=N_{4,5}(\xi)=10 \xi^{3}(1-\xi)^{2}, \\
N_{5}(\xi)=N_{5,5}(\xi)=5 \xi^{4}(1-\xi), & N_{6}(\xi)=N_{6,5}(\xi)=\xi^{5} .
\end{array}
$$




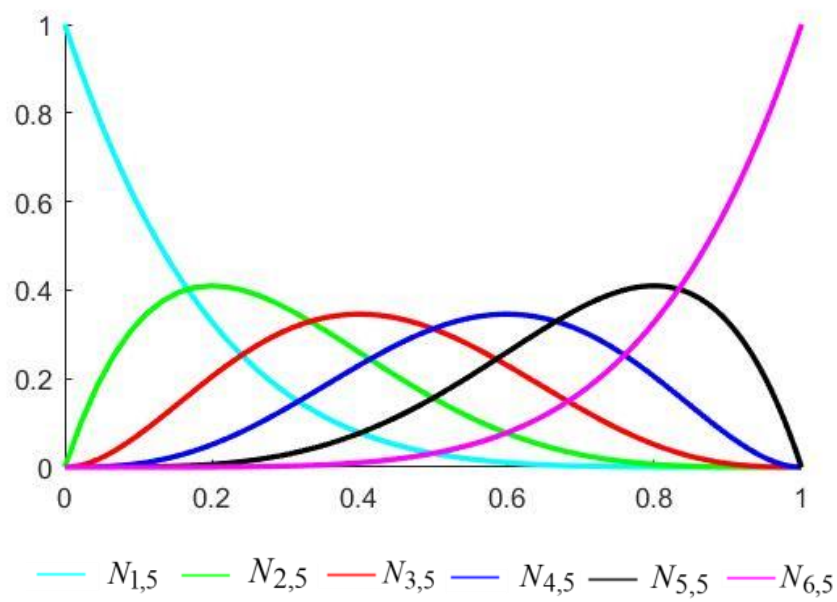

Figure 4: Quintic B-spline basis functions generated from the open knot vector $\Xi_{\xi}=[0,0,0,0,0,0,1,1,1,1,1,1]$.

Fig. 4 shows an example of a set of quintic B-Spline functions generated from an open knot vector. We can see that only the basis functions at both ends of the parametric space are interpolatory.

\subsection{Displacement function}

Bending displacement variables $v_{b}$ and geometric function are approximated by

$$
v_{b}(\xi)=\sum_{i=1}^{n} N_{i}(\xi) \hat{v}_{b i}, \quad x(\xi)=\xi L,
$$

$\hat{v}_{b i}$ as a control variable in the isogeometric analysis are equivalent to a degree of freedom in finite element analysis.

\subsection{Curvature and shear strain}

Substituting (4.6) into (2.12), we get

$$
\chi(\xi)=-\sum_{i=1}^{n} N_{i, x x}(\xi) \hat{v}_{b i}=\left\langle B_{b}\right\rangle\left\{\hat{v}_{n}\right\}, \quad\left\langle B_{b}\right\rangle=-\left\langle\begin{array}{lll}
N_{1, x x} & \cdots & N_{n, x x}
\end{array}\right\rangle_{1 x n}
$$

Substituting (4.6) into (2.13) we get

$$
\gamma(\xi)=-\frac{\phi L^{2}}{12} \sum_{i=1}^{n} N_{i, x x x}(\xi) \hat{v}_{b i}=\left\langle B_{s}\right\rangle\left\{\hat{v}_{n}\right\}, \quad\left\langle B_{s}\right\rangle=-\frac{\phi L^{2}}{12}\left\langle N_{1, x x x} \quad \cdots \quad N_{n, x x x}\right\rangle_{1 x n} .
$$

Where curvature is second derivatives of bending displacement function $v_{b}$ and the transverse shear strain is third derivatives of bending displacement function $v_{b}$. 


\subsection{Bending and shear stiffness}

From (3.16), we obtain the bending stiffness $\left[k_{b}\right]$ and shear stiffness $\left[k_{s}\right]$, respectively: For $p=3$ :

$$
\left[k_{b}\right]=\frac{3 E I}{L^{3}}\left[\begin{array}{cccc}
4 & -6 & 0 & 2 \\
-6 & 12 & -6 & 0 \\
0 & -6 & 12 & -6 \\
2 & 0 & -6 & 4
\end{array}\right], \quad\left[k_{s}\right]=\frac{3 E I}{L^{3}} \phi\left[\begin{array}{cccc}
1 & -3 & 3 & -1 \\
-3 & 9 & -9 & 3 \\
3 & -9 & 9 & -3 \\
-1 & 3 & -3 & 1
\end{array}\right]
$$

From the sum of bending and shear stiffness matrix we obtain the element stiffness matrix $[k]$ :

$$
[k]=\left[k_{b}\right]+\left[k_{s}\right]=\frac{3 E I}{L^{3}}\left[\begin{array}{cccc}
(4+\phi) & -(6+3 \phi) & 3 \phi & (2-\phi) \\
-(6+3 \phi) & (12+9 \phi) & -(6+9 \phi) & 3 \phi \\
3 \phi & -(6+9 \phi) & (12+9 \phi) & -(6+3 \phi) \\
(2-\phi) & 3 \phi & -(6+3 \phi) & (4+\phi)
\end{array}\right]
$$

For $p=5$ :

$$
\begin{gathered}
{\left[k_{b}\right]=\frac{20 E I}{7 L^{3}}\left[\begin{array}{cccccc}
20 & -30 & 4 & 3 & 2 & 1 \\
-30 & 52 & -13 & -8 & -3 & 2 \\
4 & -13 & 12 & 2 & -8 & 3 \\
3 & -8 & 2 & 12 & -13 & 4 \\
2 & -3 & -8 & -13 & 52 & -30 \\
1 & 2 & 3 & 4 & -30 & 20
\end{array}\right],} \\
{\left[k_{s}\right]=\frac{10 E I}{L^{3}} \phi\left[\begin{array}{cccccc}
6 & -15 & 10 & 0 & 0 & -1 \\
-15 & 40 & -30 & 0 & 5 & 0 \\
10 & -30 & 30 & -10 & 0 & 0 \\
0 & 0 & -10 & 30 & -30 & 10 \\
0 & 5 & 0 & -30 & 40 & -15 \\
-1 & 0 & 0 & 10 & -15 & 6
\end{array}\right] .}
\end{gathered}
$$

The element stiffness matrix $[k]$ is obtained from the sum of the bending and shear stiffness matrix:

$$
[k]=\frac{10 E I}{7 L^{3}}\left[\begin{array}{cccccc}
(40+42 \phi) & -(60+105 \phi) & (8+70 \phi) & 6 & 4 & (2-7 \phi) \\
-(60+105 \phi) & (104+280 \phi) & -(26+210 \phi) & -16 & (-6+35 \phi) & 4 \\
(8+70 \phi) & -(26+210 \phi) & (24+210 \phi) & (4-70 \phi) & -16 & 6 \\
6 & -16 & (4-70 \phi) & (24+210 \phi) & -(26+210 \phi) & (8+70 \phi) \\
4 & (-6+35 \phi) & -16 & -(26+210 \phi) & (104+280 \phi) & -(60+105 \phi) \\
(2-7 \phi) & 4 & 6 & (8+70 \phi) & -(60+105 \phi) & (40+42 \phi)
\end{array}\right] .
$$




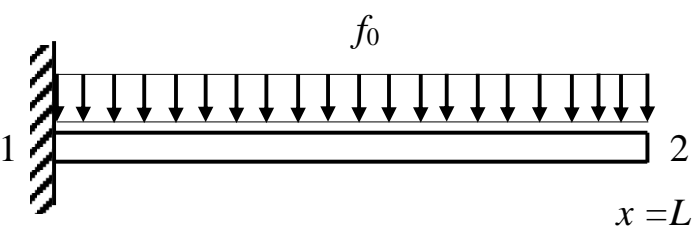

$x=0$

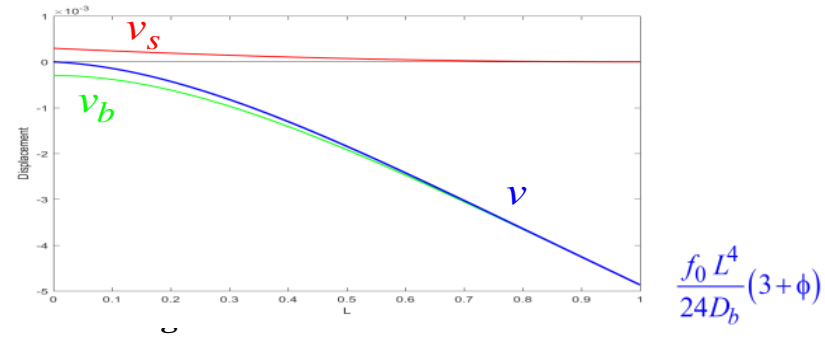

Figure 5: Fixed-Free supported beam.

Where equivalent nodal force vector

$$
\left\{f_{n}\right\}=\left\{\begin{array}{c}
\vdots \\
f_{i} \\
\vdots
\end{array}\right\}_{1, n}, \quad f_{i}=\int_{0}^{L} f\left(N_{i}-\frac{\phi L^{2}}{12} N_{i, x x}\right) \mathrm{d} x-\bar{M}\left(N_{i, x}\right)+\bar{T}\left(N_{i}-\frac{\phi L^{2}}{12} N_{i, x x}\right),
$$

For uniform distributed load $f_{0}$, the nodal force vector:

$$
\begin{aligned}
& \left\{f_{n}\right\}=\left\langle f_{n}\right\rangle^{T}=\frac{1}{4} f_{0} L\langle(1-\phi) \quad(1+\phi) \quad(1+\phi) \quad(1-\phi)\rangle^{T}, \quad(p=3), \\
& \left\{f_{n}\right\}=\left\langle f_{n}\right\rangle^{T}=\frac{1}{12} f_{0} L\langle(2-5 \phi) \quad(2+5 \phi) \quad 2 \quad 2 \quad(2+5 \phi) \quad(2-5 \phi)\rangle^{T}, \quad(p=5)
\end{aligned}
$$

\section{Numerical examination}

In this section examples with various boundary conditions for a beam under a uniform load $f_{0}$ are presented to demonstrate the performance of the UI element in static analysis. The results from Isogeometric Analysis (IGA) [21] are presented with two different order of polynomial degree $(p=3$ and $p=5)$.

\subsection{Fixed-free supported beam}

Boundary conditions: $v_{1}=0, \theta_{1}=0$. 
Table 1: Results from UI and IGA for fixed-free supported beam with 1 element.

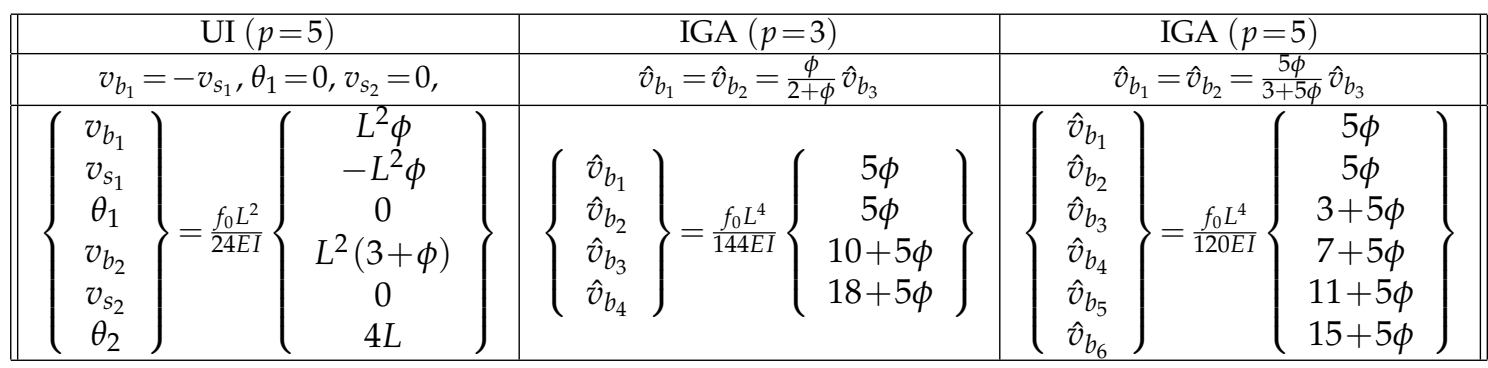

Table 2: Displacement functions of fixed-free supported beam with 1 element.

\begin{tabular}{||c|l||}
\hline Element & Displacement functions \\
\hline $\mathrm{UI}(p=5)$ & $v_{b}(x)=\frac{f_{0}}{24 E I} x\left(L^{3}-2 L x^{2}+x^{3}\right)$ \\
& $v_{s}(x)=\frac{f_{0} L^{2}}{24 E I} \phi x(L-x)$ \\
& $v(x)=\frac{f_{0}}{24 E I} x\left(x^{3}-4 L x^{2}+6 L^{2} x+\phi L^{2}(2 L-x)\right)$ \\
\hline IGA $(p=3)[21]$ & $v(x)=\frac{f_{0} L}{24 E I} x\left(5 L x-2 x^{2}+L^{2} \phi\right)$ \\
\hline IGA $(p=5)[21]$ & $v(x)=\frac{f_{0}}{24 E I} x\left(x^{3}-4 L x^{2}+6 L^{2} x+\phi L^{2}(2 L-x)\right)$ \\
\hline EXACT [21] & $v(x)=\frac{f_{0}}{24 E I} x\left(x^{3}-4 L x^{2}+6 L^{2} x+\phi L^{2}(2 L-x)\right)$ \\
\hline
\end{tabular}

Table 3: Results from UI and IGA for Simply-Simply Supported beam with 1 element.

\begin{tabular}{|c|c|c|c|c|c|c|}
\hline \multicolumn{3}{|c|}{$\mathrm{UI}(p=5)$} & IGA $(p=3)$ & \multicolumn{3}{|c|}{ IGA $(p=5)$} \\
\hline \multicolumn{3}{|c|}{$\begin{array}{l}v_{b_{1}}=-v_{s_{1}}=0 \\
v_{b_{2}}=-v_{s_{2}}=0\end{array}$} & $\begin{array}{l}\hat{v}_{b_{1}}=\frac{2 \phi}{\phi-2} \hat{v}_{b_{2}}=\frac{\phi}{2-\phi} \hat{v}_{b_{3}} \\
\hat{v}_{b_{4}}=\frac{2 \phi}{\phi-2} \hat{v}_{b_{3}}=\frac{\phi}{2-\phi} \hat{v}_{b_{2}}\end{array}$ & $\begin{array}{l}\hat{v}_{b_{1}}=\frac{1}{5} \\
\hat{v}_{b_{6}}=\frac{1}{5}\end{array}$ & $\begin{array}{l}\frac{\phi}{-3} \hat{v}_{b_{2}}=\frac{1}{3} \\
\frac{\phi}{-3} \hat{v}_{b_{5}}=\frac{1}{3}\end{array}$ & $\begin{array}{l}\bar{\delta} \phi \hat{v}_{b_{3}} \\
\bar{b} \phi \hat{v}_{b_{4}}\end{array}$ \\
\hline $\begin{array}{c}v_{b_{1}} \\
v_{s_{1}} \\
\theta_{1} \\
v_{b_{2}} \\
v_{s_{2}} \\
\theta_{2}\end{array}$ & $=\frac{f_{0} L^{3}}{24 E I}$ & $\begin{array}{c}0 \\
0 \\
1 \\
0 \\
0 \\
-1\end{array}$ & $\left.\begin{array}{l}\hat{v}_{b_{1}} \\
\hat{v}_{b_{2}} \\
\hat{v}_{b_{3}} \\
\hat{v}_{b_{4}}\end{array}\right\}=-\frac{f_{0} L^{4}}{144 E I}\left\{\begin{array}{c}\phi \\
-2+\phi \\
-2+\phi \\
\phi\end{array}\right.$ & $\begin{array}{l}\hat{v}_{b_{1}} \\
\hat{v}_{b_{2}} \\
\hat{v}_{b_{3}} \\
\hat{v}_{b_{4}} \\
\hat{v}_{b_{5}} \\
\hat{v}_{b_{6}}\end{array}$ & $=\frac{f_{0} L^{4}}{120 E I}$ & $\begin{array}{l}0 \\
1 \\
2 \\
4 \\
2 \\
0\end{array}$ \\
\hline
\end{tabular}

\subsection{Simply-simply supported beam}

Boundary conditions: $v_{1}=0, v_{2}=0$.

\subsection{Fixed-simply supported beam}

Boundary conditions: $v_{1}=0, \theta_{1}=0, v_{2}=0$. 

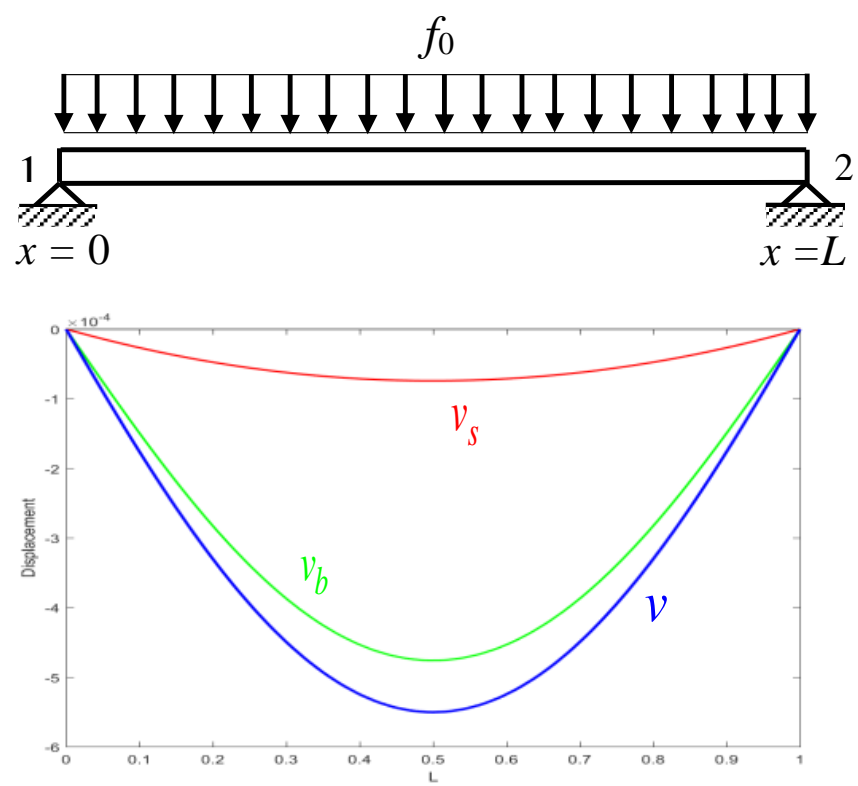

Figure 6: Simple-Simple Supported beam.

Table 4: Displacement functions of Simply-Simply supported beam with 1 element.

\begin{tabular}{||c|l||}
\hline Element & Displacement functions \\
\hline UI $(p=5)$ & $v_{b}(x)=\frac{f_{0}}{24 E I(4+\phi)}\left(\phi L^{4}+6 L^{2} x^{2}-(10 L+2 \phi L) x^{3}+(4+\phi) x^{4}\right)$ \\
& $v_{S}(x)=\frac{f_{0}}{24 E I(4+\phi)} \phi L^{2}(L-x)(4 x-L+\phi x)$ \\
& $v(x)=\frac{f_{0}}{24 E I} x\left(x^{3}-2 L x^{2}+L^{3}+L^{2} \phi(L-x)\right)$ \\
\hline IGA $(p=3)[21]$ & $v(x)=\frac{f_{0} L^{2}}{24 E I} x(L-x)$ \\
\hline IGA $(p=5)[21]$ & $v(x)=\frac{f_{0}}{24 E I} x\left(x^{3}-2 L x^{2}+L^{3}+L^{2} \phi(L-x)\right)$ \\
\hline EXACT [21] & $v(x)=\frac{f_{0}}{24 E I} x\left(x^{3}-2 L x^{2}+L^{3}+L^{2} \phi(L-x)\right)$ \\
\hline
\end{tabular}

Table 5: Results from UI and IGA for Fixed-Simply Supported beam with 1 element.

\begin{tabular}{|c|c|c|c|c|c|c|c|}
\hline \multicolumn{3}{|c|}{$\mathrm{UI}(p=5)$} & \multicolumn{2}{|c|}{ IGA $(p=3)$} & \multicolumn{3}{|c|}{ IGA $(p=5)$} \\
\hline \multicolumn{3}{|c|}{$\begin{array}{c}v_{b_{1}}=-v_{s_{1}} \theta_{1}=0 \\
v_{b_{2}}=-v_{s_{2}}=0\end{array}$} & \multicolumn{2}{|c|}{$\begin{array}{c}\hat{v}_{b_{1}}=\hat{v}_{b_{2}}=\frac{\phi-2}{4+\phi} \hat{v}_{b_{4}} \\
\hat{v}_{b_{3}}=\frac{-4+\phi^{2}}{(4+\phi) \phi} \hat{v}_{b_{4}}\end{array}$} & \multicolumn{3}{|c|}{$\begin{array}{c}\hat{v}_{b_{1}}=\hat{v}_{b_{2}}=\frac{5 \phi}{3+5 \phi} \hat{v}_{b_{3}} \\
\hat{v}_{b_{6}}=\frac{10 \phi}{5 \phi-3} \hat{v}_{b_{5}}=\frac{5 \phi}{3-5 \phi} \hat{v}_{b_{4}}\end{array}$} \\
\hline $\begin{array}{c}v_{b_{1}} \\
v_{s_{1}} \\
\theta_{1} \\
v_{b_{2}} \\
v_{s_{2}} \\
\theta_{2}\end{array}$ & $=\frac{f_{0} L^{2}}{24 E I(4+\phi)}$ & $\begin{array}{c}L^{2} \phi \\
-L^{2} \phi \\
0 \\
0 \\
0 \\
-2 L(1+\phi)\end{array}$ & $\left\{\begin{array}{l}\hat{v}_{b_{1}} \\
\hat{v}_{b_{2}} \\
\hat{v}_{b_{3}} \\
\hat{v}_{b_{4}}\end{array}\right\}=-\frac{f_{0} L^{4}}{144 E I}$ & $\begin{array}{c}\frac{(-2+\phi) \phi}{4+\phi} \\
\frac{(-2+\phi) \phi}{4+\phi} \\
\frac{\left(-4+\phi^{2}\right)}{4+\phi} \\
\phi\end{array}$ & $\begin{array}{l}\hat{v}_{b_{1}} \\
\hat{v}_{b_{2}} \\
\hat{v}_{b_{3}} \\
\hat{v}_{b_{4}} \\
\hat{v}_{b_{5}} \\
\hat{v}_{b_{6}}\end{array}$ & $=\frac{f_{0} L^{4}}{240 E I(4+\phi)}$ & $\begin{array}{c}10 \phi \\
10 \phi \\
6+10 \phi \\
8+8 \phi \\
4+4 \phi \\
0\end{array}$ \\
\hline
\end{tabular}



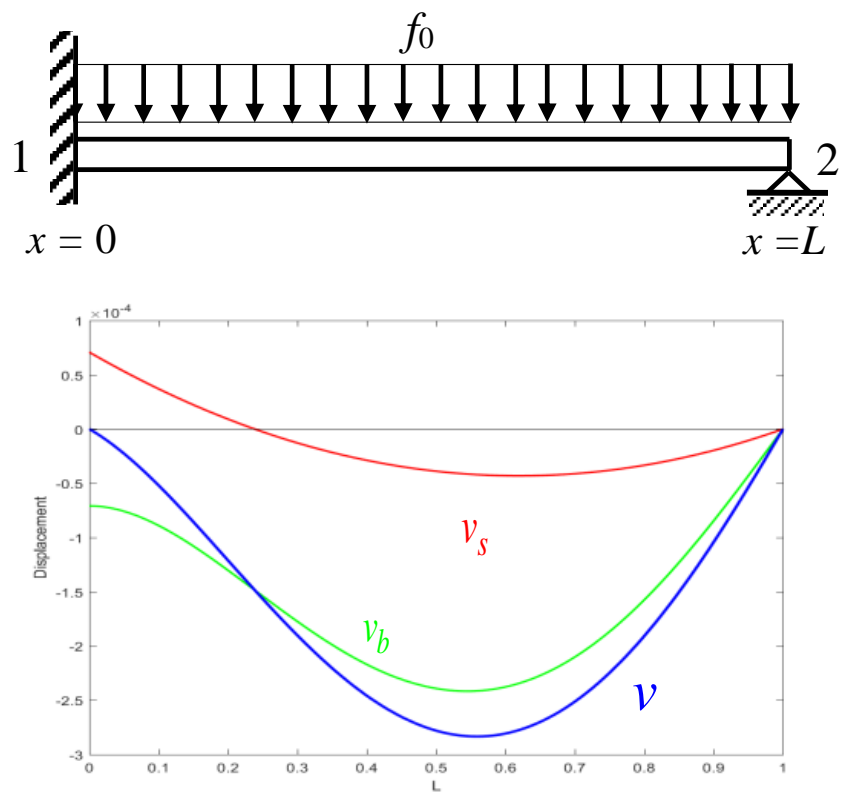

Figure 7: Fixed-Simply Supported beam.

Table 6: Displacement functions of Fixed -Simple Supported beam with 1 element.

\begin{tabular}{||c|l||}
\hline Element & Displacement functions \\
UI $(p=5)$ & $v_{b}(x)=\frac{f_{0}}{24 E I(4+\phi)}\left(\phi L^{4}-10 L x^{3}+\phi x^{4}+4 x^{4}+6 L^{2} x^{2}-2 \phi L x^{3}\right)$ \\
& $v_{S}(x)=\frac{f_{0}}{24 E I(4+\phi)} \phi L^{2}(L-x)(4 x-L+\phi x)$ \\
& $v(x)=\frac{f_{0}}{24 E I(4+\phi)} x(L-x)\left(L x(6+\phi)-x^{2}(4+\phi)+L^{2} \phi(5+\phi)\right)$ \\
\hline IGA $(p=3)[21]$ & $v(x)=\frac{f_{0} L}{24 E I(4+\phi)} x(L-x)(2 x+L \phi)$ \\
\hline IGA $(p=5)[21]$ & $v(x)=\frac{f_{0}}{24 E I(4+\phi)} x(L-x)\left(L x(6+\phi)-x^{2}(4+\phi)+L^{2} \phi(5+\phi)\right)$ \\
\hline EXACT [21] & $v(x)=\frac{f_{0}}{24 E I(4+\phi)} x(L-x)\left(L x(6+\phi)-x^{2}(4+\phi)+L^{2} \phi(5+\phi)\right)$ \\
\hline
\end{tabular}

\subsection{Simply-fixed roll supported beam}

Boundary conditions: $v_{1}=0, \theta_{2}=0$.

\subsection{Fixed-fixed roll supported beam}

Boundary conditions: $v_{1}=0, \theta_{1}=0, \theta_{2}=0$.

\subsection{Fixed-fixed supported beam}

Boundary conditions: $v_{1}=0, \theta_{1}=0, v_{2}=0, \theta_{2}=0$. 


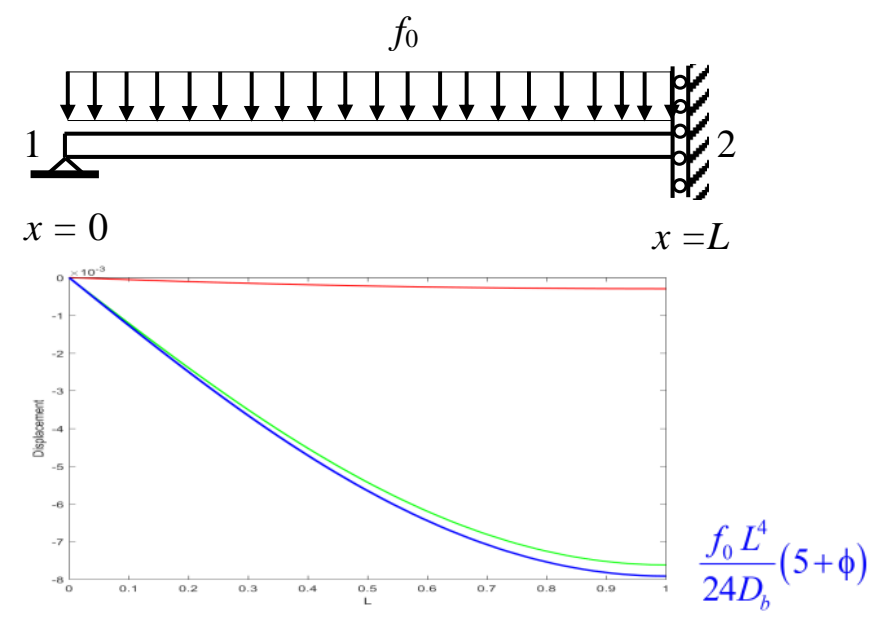

Figure 8: Simple-Fixed Roll supported beam.

Table 7: Results from UI and IGA for Simply-Fixed Roll Supported beam with 1 element.

\begin{tabular}{|c|c|c|c|c|c|c|c|}
\hline \multicolumn{3}{|c|}{$\mathrm{UI}(p=5)$} & \multicolumn{2}{|c|}{ IGA $(p=3)$} & \multicolumn{3}{|c|}{ IGA $(p=5)$} \\
\hline$v_{b_{1}}=$ & $-v_{s_{1}}=0$ & $\theta_{2}=0$ & $\hat{v}_{b_{1}}=\frac{2 \phi}{\phi-2} \hat{v}_{b_{2}}=\frac{\phi}{2-\phi}$ & $\hat{v}_{b_{3}}, \hat{v}_{b_{3}}=\hat{v}_{b_{4}}$ & $\hat{v}_{b_{1}}=\frac{10 \phi}{5 \phi-3} \hat{v}_{b}$ & $=\frac{5 \phi}{3-5 \phi}$ & $b_{3}, \hat{v}_{b_{5}}=\hat{v}_{b_{6}}$ \\
\hline$\left\{\begin{array}{c}v_{b_{1}} \\
v_{s_{1}} \\
\theta_{1} \\
v_{b_{2}} \\
v_{s_{2}} \\
\theta_{2}\end{array}\right)$ & $=\frac{f_{0} L^{2}}{24 E I}$ & $\begin{array}{c}0 \\
0 \\
8 L \\
5 L^{2} \\
\phi L^{2} \\
0\end{array}$ & $\left\{\begin{array}{l}\hat{v}_{b_{1}} \\
\hat{v}_{b_{2}} \\
\hat{v}_{b_{3}} \\
\hat{v}_{b_{4}}\end{array}\right\}=-\frac{f_{0} L^{4}}{144 E I}$ & $\left\{\begin{array}{c}\phi \\
-16+\phi \\
-30+\phi \\
-30+\phi\end{array}\right)$ & $\left\{\begin{array}{l}\hat{v}_{b_{1}} \\
\hat{v}_{b_{2}} \\
\hat{v}_{b_{3}} \\
\hat{v}_{b_{4}} \\
\hat{v}_{b_{5}} \\
\hat{v}_{b_{6}}\end{array}\right\}$ & $=\frac{f_{0} L^{4}}{120 E I}$ & $\left\{\begin{array}{c}0 \\
8 \\
16 \\
22 \\
25 \\
25\end{array}\right\}$ \\
\hline
\end{tabular}

Table 8: Displacement functions of Simply-Fixed Roll supported beam with 1 element.

\begin{tabular}{||c|l||}
\hline Element & Displacement functions \\
\hline UI $(p=5)$ & $v_{b}(x)=\frac{f_{0}}{72 E I}\left(2 \phi L^{4}+12 L^{2} x^{2}-12 L x^{3}+3 x^{4}\right)$ \\
& $\begin{array}{l}v_{S}(x)=-\frac{f_{0}}{72 E I} \phi L^{2}\left(2 L^{2}-6 L x+3 x^{2}\right) \\
v(x)=\frac{f_{0}}{24 E I} x\left(x^{3}-4 L x^{2}+8 L^{3}+L^{2} \phi(2 L-x)\right)\end{array}$ \\
\hline IGA $(p=3)[21]$ & $v(x)=\frac{f_{0} L}{24 E I} x\left(8 L^{2}-L x-2 x^{2}+L^{2} \phi\right)$ \\
\hline IGA $(p=5)[21]$ & $v(x)=\frac{f_{0}}{24 E I} x\left(x^{3}-4 L x^{2}+8 L^{3}+L^{2} \phi(2 L-x)\right)$ \\
\hline EXACT [21] & $v(x)=\frac{f_{0}}{24 E I} x\left(x^{3}-4 L x^{2}+8 L^{3}+L^{2} \phi(2 L-x)\right)$ \\
\hline
\end{tabular}

Only UI $(p=5)$ and IGA $(p=5)$ give an exact displacement function. 


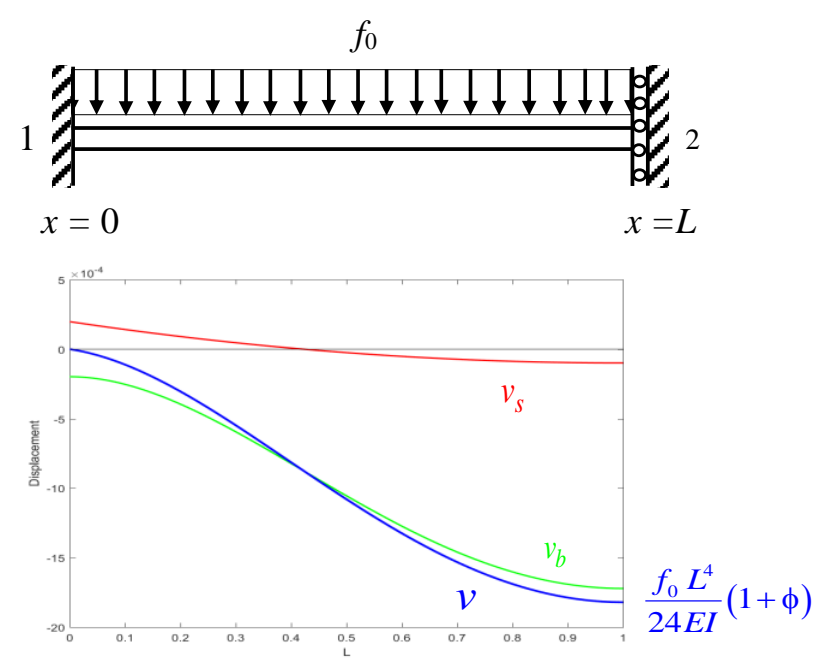

Figure 9: Fixed-Fixed Roll supported beam.

Table 9: Results from UI and IGA for Fixed-Fixed Roll Supported beam with 1 element.

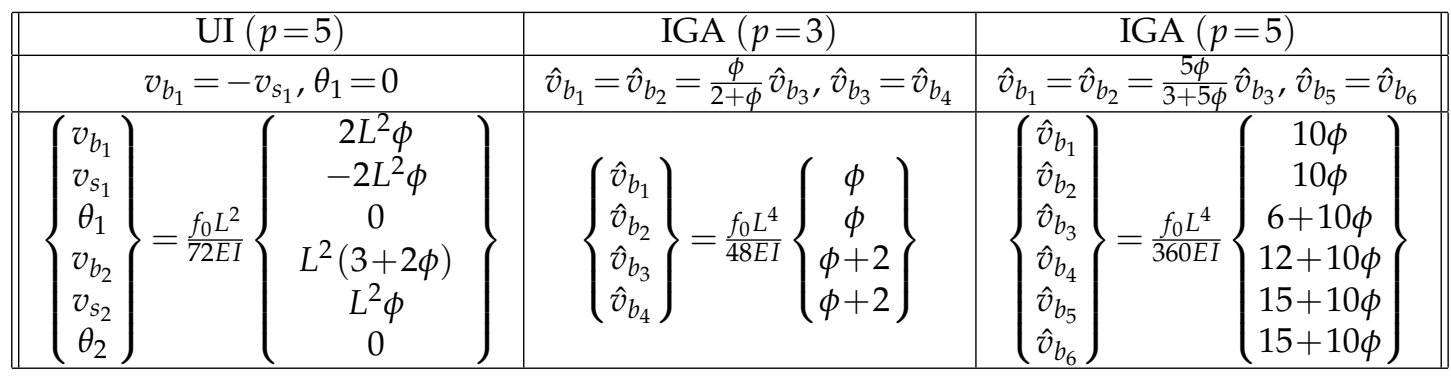

Table 10: Displacement functions of Fixed-Fixed Roll supported beam with 1 element.

\begin{tabular}{||c|l||}
\hline Element & Displacement functions \\
\hline \multirow{2}{*}{$\mathrm{UI}(p=5)$} & $v_{b}(x)=\frac{f_{0}}{72 E I}\left(2 \phi L^{4}+12 L^{2} x^{2}-12 L x^{3}+3 x^{4}\right)$ \\
& $v_{s}(x)=-\frac{f_{0}}{72 E I} \phi L^{2}\left(2 L^{2}-6 L x+3 x^{2}\right)$ \\
& $v(x)=\frac{f_{0}}{24 E I} x\left(x^{3}-4 L x^{2}+4 L^{2} x+L^{2} \phi(2 L-x)\right)$ \\
\hline IGA $(p=3)[21]$ & $v(x)=-\frac{f_{0} L}{24 E I} x\left(3 L x-2 x^{2}+L^{2} \phi\right)$ \\
\hline IGA $(p=5)[21]$ & $v(x)=\frac{f_{0}}{24 E I} x\left(x^{3}-4 L x^{2}+4 L^{2} x+L^{2} \phi(2 L-x)\right)$ \\
\hline EXACT [21] & $v(x)=\frac{f_{0}}{24 E I} x\left(x^{3}-4 L x^{2}+4 L^{2} x+L^{2} \phi(2 L-x)\right)$ \\
\hline
\end{tabular}

\section{Conclusions}

In this paper, the performance of UI and IGA Galerkin elements based on the unified and integrated method are compared. In the unified and integrated method, the total 


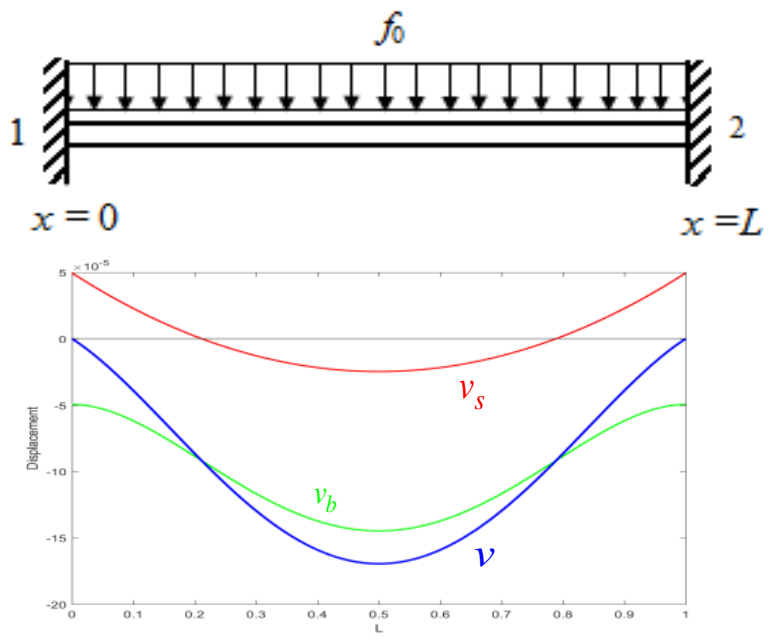

Figure 10: Fixed-fixed supported beam.

Table 11: Results from UI and IGA for Fixed-Fixed Supported beam with 1 element.

\begin{tabular}{||c|c|c||}
\hline UI $(p=5)$ & IGA $(p=3)$ & IGA $(p=5)$ \\
\hline$v_{b_{1}}=-v_{s_{1}}, v_{b_{2}}=-v_{s_{2}}$ & $\hat{v}_{b_{1}}=\hat{v}_{b_{2}}=\hat{v}_{b_{3}}=\hat{v}_{b_{4}}=0$ & $\hat{v}_{b_{1}}=\hat{v}_{b_{2}}=\frac{5 \phi}{3+5 \phi} \hat{v}_{b_{3}}, \hat{v}_{b_{5}}=\hat{v}_{b_{6}}=\frac{5 \phi}{3+5 \phi} \hat{v}_{b_{4}}$ \\
\hline $\left.\begin{array}{c}L^{2} \phi \\
v_{b_{1}} \\
v_{s_{1}} \\
\theta_{1} \\
v_{b_{2}} \\
v_{s_{2}} \\
\theta_{2}\end{array}\right\}=\frac{f_{0} L^{2}}{144 E I}\left\{\begin{array}{r}L^{2} \phi \\
-L^{2} \phi \\
0\end{array}\right\}$ & $\left\{\begin{array}{l}\hat{v}_{b_{1}} \\
\hat{v}_{b_{2}} \\
\hat{v}_{b_{3}} \\
\hat{v}_{b_{4}}\end{array}\right\}=\left\{\begin{array}{l}0 \\
0 \\
0 \\
0\end{array}\right\}$ & $\left.\begin{array}{l}10 \phi \\
10 \phi \\
6+10 \phi \\
\hat{v}_{b_{2}} \\
\hat{v}_{b_{3}} \\
\hat{v}_{b_{4}} \\
\hat{v}_{b_{5}} \\
\hat{v}_{b_{6}}\end{array}\right\}=\frac{f_{0} L^{4}}{1440 E I}\left\{\begin{array}{c}6+10 \phi \\
10 \phi \\
10 \phi\end{array}\right\}$ \\
\hline
\end{tabular}

Table 12: Displacement functions of Fixed -Fixed supported beam with 1 element.

\begin{tabular}{||c|l||}
\hline Element & Displacement functions \\
\hline \multirow{3}{*}{ UI $(p=5)$} & $v_{b}(x)=\frac{f_{0}}{144 E I}\left(6 L^{2} x^{2}-12 L x^{3}+6 x^{4}+\phi L^{4}\right)$ \\
& $v_{S}(x)=\frac{f_{0}}{144 E I} \phi L^{2}\left(L^{2}-6 L x+6 x^{2}\right)$ \\
& $v(x)=\frac{f_{0}}{24 E I} x\left(x^{3}-2 L x^{2}+L^{2} x+L^{2} \phi(L-x)\right)$ \\
\hline IGA $(p=3)[21]$ & $v(x)=0$ \\
\hline IGA $(p=5)[21]$ & $v(x)=\frac{f_{0}}{24 E I} x\left(x^{3}-2 L x^{2}+L^{2} x+L^{2} \phi(L-x)\right)$ \\
\hline EXACT [21] & $v(x)=\frac{f_{0}}{24 E I} x\left(x^{3}-2 L x^{2}+L^{2} x+L^{2} \phi(L-x)\right)$ \\
\hline
\end{tabular}

displacement is split into bending displacement and shear displacement, which is the key to combine Bernoulli and Timoshenko theory. The weak form differential equation or principal virtual work (PVW), that is the symmetric form of the second-order derivative for the bending part and the third order derivative for the shear part, gives completely 
locking-free results.

Conclusions drawn from the results of the numerical examination are:

1. The formulation of the shear deformable UI element and IGA Galerkin $(p=5)$ can give exact solutions not only at nodes but also along the beam, proving that it is free from shear locking, which is due to the strong relationship between shear displacement, rotation, curvature, transverse shear.

2. IGA Galerkin $(p=3)$ element only give exact DOFs values at nodes.

3. Since $f_{0}$ is constant, the analytical solution is of fourth order in all cases. Therefore, all results presented by only one element indicate that UI $(p=5)$ and IGA Galerkin $(p=5)$ are able to give exact DOFs values and displacement functions.

4. When this element is applied to the thin beam case, i.e., $\phi=0$, all equations will automatically be transformed into Bernoulli theory in which the total displacement is equal to bending displacement.

\section{Acknowledgements}

The research support from the Ministry of Research and Technology/National Research and Inovation Agency (RISTEK-BRIN), Indonesia, through the PDUPT program (Grant No. NKB-1641/UN2.R3.1/HKP.05.00/2019) is gratefully acknowledged.

\section{References}

[1] S. TIMOSHENKO, On the correction for shear of differential equation for transverse vibrations of prismatic bars, Philosophical Magazine, 41 (1921), pp. 744-746.

[2] S. Timoshenko, On the transverse vibrations of bars of uniform cross section, Philosophical Magazine, 43 (1922), pp. 125-131.

[3] G. PRATHAP AND G. BHASHYAM, Reduced integration and the shear-flexible beam element, Int. J. Numer. Methods Eng., 18 (1982), pp. 172-178.

[4] K.-U. BLETZINGER, M. BISCHOFF AND E. RAMM, A unified approach for shear-locking-free triangular and rectangular shell finite elements, Computers and Structures, 75 (2000), pp. 321334.

[5] J. L. Batoz and G. Dhatt, Modélisation des Structures par éléments Finis : Poutres et Plaques, Vol. 2, Hermes Science Publications, 1990.

[6] I. KAtiLI, Unified and integrated approach in a new Timoshenko beam element, Euro. J. Comput. Mech., 26 (2017), pp. 282-308.

[7] I. Katili, A new discrete Kirchhoff-Mindlin element based on Mindlin-Reissner plate theory and assumed shear strain fields, part I: An extended DKT element for thick-plate bending analysis, Int. J. Numer. Methods Eng., 36 (1993), pp. 1859-1883.

[8] I. KATILI, A new discrete Kirchhoff-Mindlin element based on Mindlin-Reissner plate theory and assumed shear strain fields, part II: An extended DKQ element for thick plate bending analysis, Int. J. Numer. Methods Eng., 36 (1993), pp. 1885-1908. 
[9] I. Katili, J. L. Batoz, I. J. MaKnun, A. Hamdouni And O. Millet, The development of $D K M Q$ plate bending element for thick to thin shell analysis based on naghdi/reissner/mindlin shell theory, Finite Elements in Analysis and Design, 100 (2015), pp. 12-27.

[10] I. Katili, I. J. MAKnUn, A. HAMDOUni AND O. Millet, Application of DKMQ element for Composite plate bending structures, Composite Structures, 132 (2015), pp. 166-174.

[11] I. J. MAKNUn, I. KaTILI, O. Millet AND A. HAMDOUnI, Application of DKMQ element for twist of thin-walled beams: comparison with Vlasov theory, Int. J. Comput. Methods Eng. Sci. Mech., 17 (2016), pp. 391-400.

[12] I. KATILI, J. L. BATOZ, I. J. MAKNUN AND P. LARDEUR, A comparative formulation of DKMQ, DSQ and MITC4 quadrilateral plate elements with new numerical results based on s-norm tests, Computer and Structures, 204 (2018), pp. 48-64.

[13] I. Katili, I. J. MAKNun, J. L. BATOZ AND A. IbRAhimbegović, Shear deformable shell element DKMQ24 for composite structures, Composite Structures, 202 (2018), pp. 182-200.

[14] I. Katili, I. J. MaKnUn, J. L. BAtOz AND A. M. Katili, Asymptotic equivalence of DKMT and MITC3 elements for thick composite plates, Composite Structures, 206 (2018), pp. 363-379.

[15] H. IRPANNI, I. KATILI AND I. J. MAKNUN, Development DKMQ shell element with five degrees of freedom per nodal, Int. J. Mech. Eng. Robot. Res., 6 (2017), pp. 248-252.

[16] I. KATILI, I. J. MAKNUN AND E. TJAHJONO, Alisyahbana I. Error estimation for the DKMQ24 shell element by using various recovery methods, Int. J. Tech., 6 (2017), pp. 1060-1069.

[17] F. T. WONG, ERWIN RICHARD A AND I. KATILI, Development of the DKMQ element for buckling analysis of shear-deformable plate bending, Proc. Eng., 171 (2017), pp. 805-812.

[18] I. SENJANOVIĆ, N. VladimiR, M. TOMIĆ, An advanced theory of moderately thick plate vibrations, J. Sound Vibration, 332 (2013a), pp. 1868-1880.

[19] H. T. Thai, T. K. NGUYen, T. P. Vo AND T. NGO, A new simple shear deformation plate theory, Composite Structures, 171 (2017), pp. 277-285.

[20] I. KATILI AND R. ARISTIO, Isogeometric Galerkin in rectangular plate bending problem based on UI approach, Euro. J. Mech. A Solids, 67 (2018), pp. 92-107.

[21] J. Kiendl, F. Auricchio, T. J. R. Hughes AND A. ReAli, Single-variable formulations and isogeometric discretization for shear deformable beams, Computer Methods Appl. Mech. Eng., 284 (2015), pp. 988-1004.

[22] K. KAPUR, Vibrations of a Timoshenko beam using finite-element approach, J. Acoustic Soc. Am., 40 (1966), pp. 1058-1063.

[23] X. F. LI, A unified approach for analyzing static and dynamic behavior of functionally graded Timoshenko and Bernoulli-Euler beam, J. Sound Vibration, 318 (2008), pp. 1210-1229.

[24] G. FAlsone AND D. SetTineRI, An Bernoulli-Euler-like finite element method for Timoshenko beams, Mech. Res. Commun., 38 (2011), pp. 12-16.

[25] I. Katili, T. SYAhril AND A. M. KaTILI, Static and free vibration analysis of FGM beam based on unified and integrated of Timoshenko's theory, Composite Structures, 242 (2020), https://doi.org/10.1016/j. compstruct.2020.112130.

[26] C. DE BOoR, A Practical Guide to Splines (revised edition), Springer, 2001. 